\title{
Effects of the Coordinates Planes Crystal Orientation on the Structural Strength of Single-Crystal Turbine Vanes and Blades*
}

\author{
Jinxiang CHEN**, Ryosaku HASHIMOTO***, Yoshitaka FUKUYAMA***, \\ Masahiro MATSUSHITA***, Akinori OGAWA***, Makoto OSAWA****, \\ Tadaharu YOKOKAWA**** and Hiroshi HARADA**** \\ ** Aeronautical Environment technology Center, Japan Aerospace Exploration Agency, \\ 7-44-1 Jindaiji, Chofu, 182-8522 Japan; Present workplace: Institute of Industrial Science, \\ the University of Tokyo, 4-6-1 Komaba, Meguro-ku, Tokyo 153-8505 Japan \\ E-mail:chenj@fsis.iis.u-tokyo.ac.jp \\ ${ }^{* * \star}$ Aeronautical Environment technology Center, Japan Aerospace Exploration Agency \\ **** Materials Engineering laboratory, High Temperature Materials Group, \\ National Institute for Materials Science, 1-2-1 Sengen, Tsukuba, 305-0047 Japan
}

\begin{abstract}
The effects of crystal orientation $(\theta)$ on the structural strength of single crystal turbine vanes and blades calculated with the finite element method (FEM) are discussed in this paper. TMS-75, a $3^{\text {rd }}$ generation single-crystal Ni-base superalloy, is chosen as the model material for turbine vanes and blades. It became clear that, (1) the elastic constant matrix changes were equivalence for each of three coordinate due to the orientation variation $\left(0^{\circ}<\theta<90^{\circ}\right)$, and the strength of the turbine vane and blade were strongly related to $\theta$, and also depended on the load and model shape. (2) The strength dependence of the turbine vane on the crystal orientation was depended on coordinate plane: there are lower Mises stress in XY plane and maximum Mises stress in near the $\theta=45^{\circ}$ at both $\mathrm{YZ}$ and ZX Planes. (3) In the case of a blade, the influence is similar to the vane on blade tip, but the converse holds for the blade root. It is clear that the creep rupture time can be extended, when the $<100>$ crystallographic axes is the $\mathrm{Y}$ or $\mathrm{X}$ axis of the blade under higher rotation speed.
\end{abstract}

Key words: Anisotropy, Elasticity, Structural Analysis, Thermal Stress, Single-Crystal, TMS-75, Orientation

\section{Introduction}

The thermal efficiency of gas turbine is an important factor that strongly affects the $\mathrm{CO}_{2}$ emission reduction and the prevention of global warming, and in order to make significant improvements in this efficiency, the increase in the turbine inlet temperature (TIT) is required. For this reason, Ni-base single crystal (SC) superalloys were developed for the applications in gas turbines in order to achieve increasingly higher operating temperatures ${ }^{(1)}$. The SC superalloy shows the anisotropic mechanical properties. However, the in-plane crystal orientation is conventionally not controlled. The effect of elastic anisotropy is being researched through the comparison between the isotropic and the anisotropic analyses ${ }^{(2) \sim(4)}$ and thermal stress analysis of SC superalloy during growth ${ }^{(5) \sim(7)}$. Anisotropy in multiaxial creep $^{(8),}{ }^{(9)}$ and fatigue has also been investigated ${ }^{(10) \sim(12)}$. Simple models as cylinder ${ }^{(6)}$ and simple thermal loads have been reported in most published papers ${ }^{(5),(7)}$. To evaluate the structural strength of turbine blades and vanes made of SC superalloy, the anisotropies of creep and plastic deformation should be considered. However, the turbine blades and vanes have complex mechanical properties, complicated shapes, and an austere working 
environment, so, it is difficult to completely evaluate their structural strength. These factors have hindered the analysis and evaluation of the structural strength of practical turbine blades and vanes made of SC superalloy in a working environment. As SC superalloy turbine blades and vanes are expensive, the best use should be made of the anisotropy by reconciling their property with the operating environment. The structural strength characteristics and their dependency on the crystal orientation should be investigated. Under this background the anisotropies of creep deformation and plastic deformation were not considered during the initial stage of our research. Instead, we investigated the structural strength using von Mises stress as one parameter and the creep rupture time calculated from the Larson Miller curve in the $<001>$ direction as another ${ }^{(3),(13)}$. We previously estimated the change in structural strength of turbine blades and vanes due to single crystallization ${ }^{(3)}$ and investigated the effects of the cross-sectional in-plane of the XY crystal orientation on the structural strength of SC turbine vanes ${ }^{(13)}$.

In this paper, the differences in the effects of the cross-sectional $\mathrm{YZ}$ and ZX in-plane (also called $\mathrm{X}$ and $\mathrm{Y}$ axes, respectively) crystal orientations compared with those of the XY in-plane (also called $\mathrm{Z}$ axis) crystal orientation on the structural strength of blades and vanes were investigated for broad applications of the SC in the gas turbine industry.

\section{Analytical Modeling Descriptions}

The analytical model is of a 1st stage gas turbine vane and of blade made of TMS-75. Figure 1 (a) (b) show the analytical model of a turbine vane and blade. In Fig. 1, the dimensionless parameter $\mathrm{H}$ corresponds to the vane height, where the normalized height $\mathrm{H} 0 \%$ and $\mathrm{H} 100 \%$ represents the hub and tip of the turbine vane, respectively. In the same manner, the dimensionless parameter L represents the external surface position as shown in Fig. 1 (c). The leading edge and trailing edge are located at the normalized surface distance $0 \%$ and $100 \%$ positions, respectively. The pressure side was set as negative and the suction side as positive. $\mathrm{X}, \mathrm{Y}$ and $\mathrm{Z}$-axis represent the axial, circumferential and radial direction of the blade and vane respectively. As taking a sample, Fig. 1 (d) shown a case which the $\mathrm{Y}$-axis coincides with the [010] crystallographic direction, the crystal orientation in ZX Plane is described by $\theta$, the angle between SC superalloy [001] direction and Z-axis. We investigated six cases with $\theta$ ranging from 0 to $75^{\circ}$ at $15^{\circ}$ intervals. For convenience, we also use $\theta$ to describe the crystal orientations of the XY and YZ in-planes ${ }^{(13)}$. The thermal load and gas pressure were close to those in a $1400^{\circ} \mathrm{C}$ class gas turbine ${ }^{(13),(14)}$. The temperature distribution on the external surfaces of the turbine blades for different cross-sections is shown in Fig. 2. The rotational speed was $10200 \mathrm{rpm}$, and the rotation center axis, $\mathrm{Z}$, was at $-0.3367 \mathrm{~m}$. The turbine blades were constrained in the same way as previously reported $^{(3),(13)}$. Analysis was done using Nastran for Windows Visual 2004. The

(a)

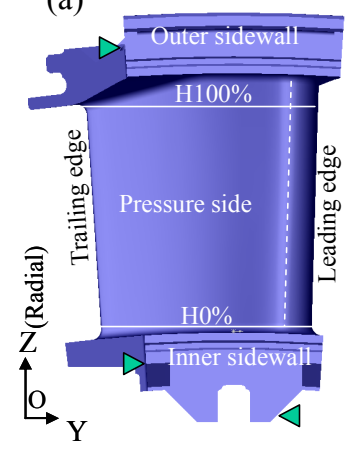

(b)

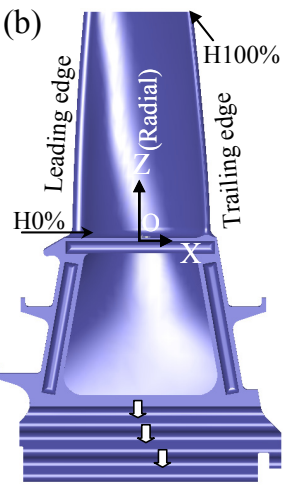

(c)

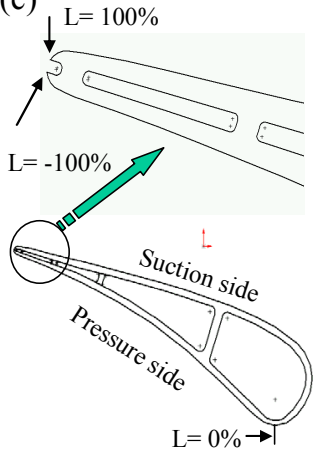

(d)

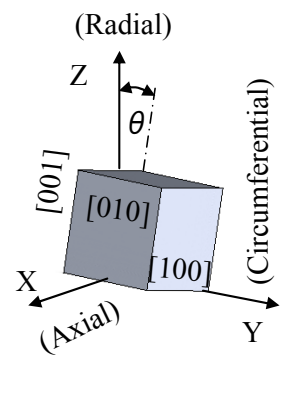

Fig. 1 Analysis model (a) vane, (b) blade and normalized position in span direction of vane (c) and single crystal direction with $\theta$ degree (d). 
blade and vane geometry was modeled by the 94,783 and 106,042 tetrahedral finite elements and 29,485 and 33,318 nodes, respectively. The creep rupture life analysis and the data processing were carried out by the evaluation system developed by the author $^{(14)}$.

\section{Formulation, FEM Results, and Discussion}

\subsection{Formulation}

For cubic SCs, when the X, Y and Z-axes coincide with the crystallographic axes (Fig. 1 (c), $\theta=0^{\circ}$ ), there are only three independent elastic constants $c_{11}, c_{12}$ and $c_{44}$ as expressed in Eq. $(1)^{(15)}$.

$$
\left\{\begin{array}{l}
\sigma_{1} \\
\sigma_{2} \\
\sigma_{3} \\
\sigma_{12} \\
\sigma_{23} \\
\sigma_{31}
\end{array}\right\}=\left[\begin{array}{cccccc}
c_{11} & c_{12} & c_{12} & 0 & 0 & 0 \\
& c_{11} & c_{12} & 0 & 0 & 0 \\
& & c_{11} & 0 & 0 & 0 \\
& & & c_{44} & 0 & 0 \\
& \text { Sym. } & & & c_{44} & 0 \\
& & & & & c_{44}
\end{array}\right]\left\{\begin{array}{c}
\varepsilon_{1} \\
\varepsilon_{2} \\
\varepsilon_{3} \\
\varepsilon_{12} \\
\varepsilon_{23} \\
\varepsilon_{31}
\end{array}\right\} \cdots(1)
$$

Here, indices 1, 2 and 3 indicate the crystallographic axes of the material (SC superalloy). For practicality, Eq. (1) is described by the Eq. (1a).

$$
\{\sigma\}_{123}=[A]\{\varepsilon\}_{123} \cdots(1 a)
$$

When $\theta \neq 0^{\circ}$ as shown in Fig. 1 (c), direction cosine from the crystallographic axes to Cartesian coordinate in ZX Plane is described as Eq. (2) ${ }^{(16)}$.

$$
g=\left[\begin{array}{ccc}
\cos \theta & 0 & -\sin \theta \\
0 & 1 & 0 \\
\sin \theta & 0 & \cos \theta
\end{array}\right] \ldots \ldots \ldots \ldots(2)
$$

In this case, the elastic constant matrix $\left[A^{\prime}\right]$ in Cartesian coordinate is determined by the coordinate transformation rule of Eq. $(3)^{(17)}$.

$$
\left[A^{\prime}\right]=[T][A][T]^{T}
$$

Here, $[T]$ is the coordinate transformation matrix, which is based on Eq. $(2)^{(18),(19)}$. Equation (4) was obtained from (1)-(3). 


$$
\left[A^{\prime}\right]=\left[\begin{array}{cccccc}
c_{11}^{\prime} & c_{12} & c_{12}^{\prime} & 0 & 0 & c_{16}^{\prime} \\
& c_{11} & c_{12} & 0 & 0 & 0 \\
& & c_{11}^{\prime} & 0 & 0 & -c_{16}^{\prime} \\
& & & c_{44} & 0 & 0 \\
& \text { Sym. } & & & c_{44} & 0 \\
& & & & & c_{44}^{\prime}
\end{array}\right] \cdots(4)
$$

with

$$
\begin{aligned}
& c_{11}^{\prime}=c_{11}-\sin ^{2} 2 \theta \cdot c \\
& c_{12}^{\prime}=c_{12}+\sin ^{2} 2 \theta \cdot c \\
& c_{44}^{\prime}=c_{44}+\sin ^{2} 2 \theta \cdot c_{-} \\
& c_{16}^{\prime}=-\sin 4 \theta \cdot c_{-} / 2 . \\
& c_{-}=\left(c_{11}-c_{12}-2 \cdot c_{44}\right) / 2 .
\end{aligned}
$$

The von Mises stress was investigated using

$$
\sigma=\sqrt{\left[\left(\sigma_{x}-\sigma_{y}\right)^{2}+\left(\sigma_{y}-\sigma_{z}\right)^{2}+\left(\sigma_{z}-\sigma_{x}\right)^{2}\right] / 2+3\left(\tau_{x y}^{2}+\tau_{y z}^{2}+\tau_{z x}^{2}\right)}
$$

Each stress component was investigated using (4), so Eq. (5) can be rewritten as Eq. (6) through Eq. (4).

$$
\sigma=\sqrt{\sigma_{0}+\sigma_{\theta}}
$$

with

$$
\begin{aligned}
& \sigma_{0}=\left(c_{11}-c_{12}\right)^{2} \varepsilon_{a} / 2+3 c_{44}^{2} \varepsilon_{b} \\
& \varepsilon_{a}=\left(\varepsilon_{y}-\varepsilon_{z}\right)^{2}+\left(\varepsilon_{x}-\varepsilon_{z}\right)^{2}+\left(\varepsilon_{x}-\varepsilon_{y}\right)^{2} \cdots(7 a) \\
& \varepsilon_{b}=\varepsilon_{x y}^{2}+\varepsilon_{y z}^{2}+\varepsilon_{z x}^{2} \\
& \sigma_{\theta}=3 c_{+} c_{-}\left(\varepsilon_{c} \sin ^{2} 2 \theta+\varepsilon_{d} \sin 4 \theta\right) \\
& \varepsilon_{c}=\varepsilon_{z x}^{2}-\left(\varepsilon_{x}-\varepsilon_{z}\right)^{2} \\
& \varepsilon_{d}=\left(\varepsilon_{x}-\varepsilon_{z}\right) \varepsilon_{z x} \\
& c_{+}=\left(c_{11}-c_{12}+2 c_{44}\right) / 2
\end{aligned}
$$

These similar to equations used for the ZX in-plane ${ }^{(13)}$. The result is also applied to equations of Mises stress for the YZ in-plane. The elastic constant matrix changes were equivalent for the three coordinate planes due to the differences in orientation $(\theta)$, as represented by Eqs. (4a)-(4e). From Eqs. (6) to (8), $\sigma$ can be expressed as $\sigma_{\theta}$, which depends on $\theta$ and $\sigma_{0}$, which does not depend on $\theta$ formally. However, the $\sigma_{0}$ related to each strain depends on $\theta$. Therefore, $\sigma$ is not only dependent on $\theta$, it is affected by many factors such as the elastic constant of the material, the thermal environment, the gas pressure, and the model shape. The FEM method was used to analyze the effects of $\theta$ on 
the structural strength due to the complex shapes and loads of the blades and vanes.

\subsection{Results and discussion}

\subsubsection{Von Mises stress}

We first investigate the stress variation in a turbine vane with $\theta$ in the range $0^{\circ}$ to $90^{\circ}$. Figure 3 (a) shows the average stress at 101 points uniformly located on the external surfaces of the vane for three different cross-sections: $\mathrm{H} 10 \%, \mathrm{H} 50 \%$, and $\mathrm{H} 90 \%$. There was one case in which the average stress of each cross-section for the XY in-plane was the smallest and most equable. However, there were also cases in which the average stress for the $\mathrm{YZ}$ and $\mathrm{ZX}$ in-planes strongly depended on $\theta$, and the maximum Mises stress was near $\theta=45^{\circ}$ for both the $\mathrm{YZ}$ and $\mathrm{ZX}$ in-planes. The maximum stress was more than $30 \%$ higher than that of average stress of the XY in-plane for each cross-section.

To facilitate discussion of stress at a partial position (nodal stress), we plot in Figs. 3 (b) and (c) the relationship between nodal stress and $\theta$ for the positions on the leading edge ( $\mathrm{L}=0 \%$ ) that cross $\mathrm{H} 90 \%$ and $\mathrm{H} 50 \%$ and for a few other positions $(\mathrm{L}= \pm 48 \%$ and $96 \%$ ) for the turbine vanes and blades.

We found that, compared with the average stress, the nodal stress had a larger change region and strongly depended on $\theta$. However, the relationships between the stress at the most nodes and $\theta$ were the same as those between the average stress and $\theta$.

The strength of an SC superalloy turbine vane is affected by the crystal orientation of the XY in-plane, and these affects cannot be ignored $^{(13)}$. However, our current findings clearly show that the crystal orientation of the XY plane has less effect on the strength than those of the YZ and ZX in-planes. For actual vanes, the control of the [001] orientation in the radial direction (the XY in-plane) is a well-established manufacturing method.

Therefore, the control method is appropriate under present manufacturing technology.

We next investigate the turbine blades. The results were different than those for the turbine vanes because the blades were subjected to
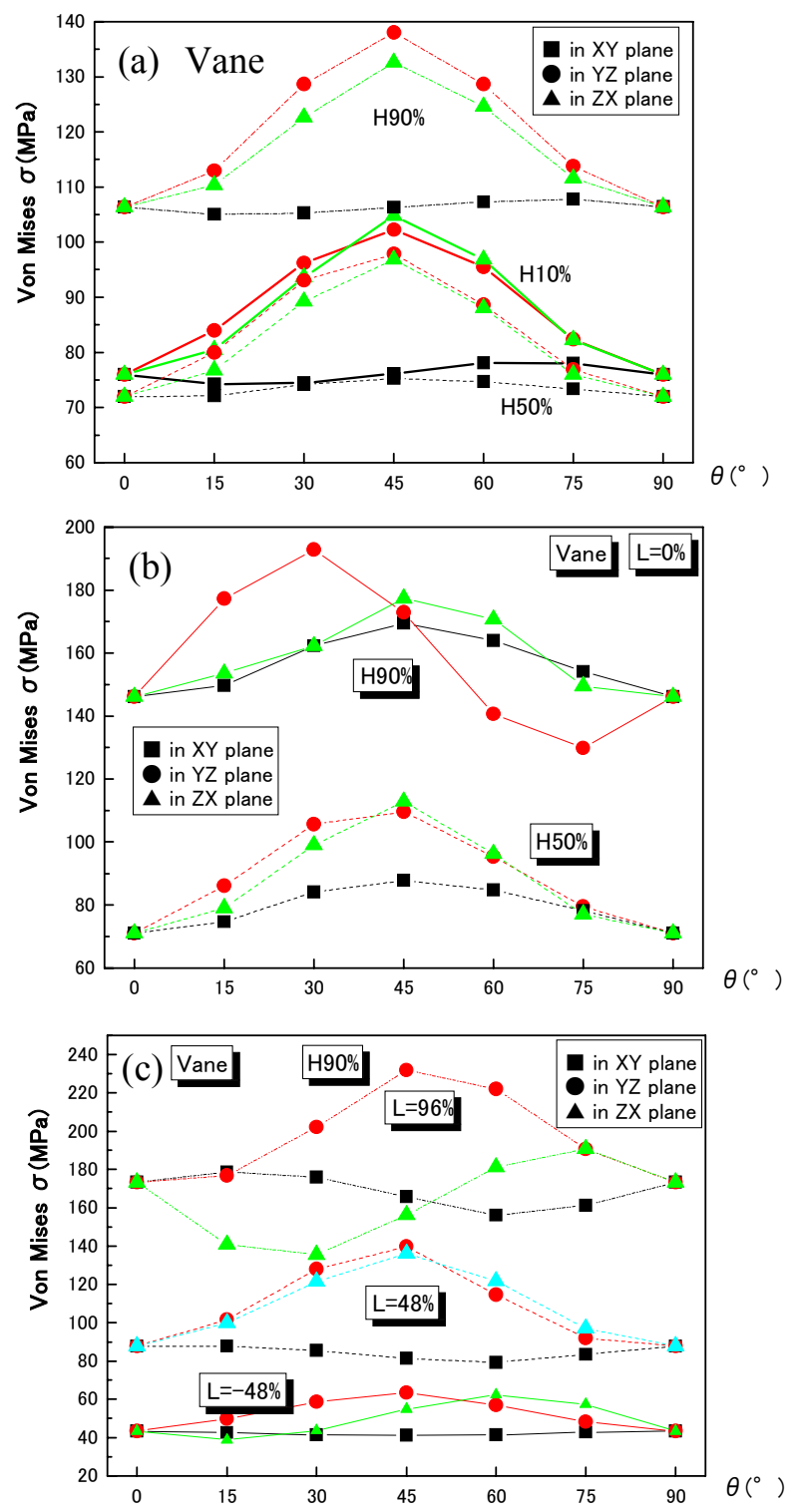

Fig. 3 Relationship between the Mises stress and $\theta$ for three axis of vane at the different spanwise cross section (a) and nodes (b), (c). 
centrifugal force. The distributions of the Mises stress for the blades are shown in Figs. 4 (a)-(c). The cross-section used in (b) and (c) was H10\%, not the H90\% used in Figs. 3. (b) and (c). Because the stress of a blade root made of SC superalloy is higher than that made of conventional casting alloy, the blade root is a critical area and should be discussed. It is clear that relationship between $\theta$ and average stress on the external surfaces of the turbine blade differs between the cross-sections. Because of the low centrifugal force on the blade, the change pattern of the Mises stress was similar to that on the vane for the H90\% cross-section, while with larger centrifugal stress, the change pattern was different to that on the blade for the H10\% cross-section. That is, there was maximum Mises stress for the XY in-plane (Fig. 4(a) $\boldsymbol{\Delta}$ ) and minimum Mises stress near $\theta=45^{\circ}$ and $30^{\circ}$ in the $\mathrm{YZ}$ and ZX in-planes, respectively. With moderate centrifugal stress of the $\mathrm{H} 50 \%$ cross-section, there was not an extreme value of Mises stress near $\theta=45^{\circ}$ for each in-plane. The Mises stress for the YZ and ZX in-planes varied with $\theta$, unlike for the XY in-plane.

The relationship between nodal stress and $\theta$ for the positions on the leading edge $(\mathrm{L}=0 \%)$ that intersect the $\mathrm{H} 10 \%$ and $\mathrm{H} 50 \%$ cross-sections of the turbine blade are shown in Fig. 4 (b). The change region of the nodal stress was larger than that of the average stress. However, the relationship between stress and $\theta$ was the same. For other positions, the converse was true, such as for the $\mathrm{H} 10 \%$ and $\mathrm{H} 50 \%$ intersection positions. In short, the Mises stress is affected by the load, the model shape, and $\theta$-it shows complex mechanical properties.

However, there are differences between the relationships for the $\mathrm{YZ}$ and $\mathrm{ZX}$ in-planes and those for the XY in-plane. For example, for the XY in-plane, $\sigma_{\theta}$ depended on the $\varepsilon_{z}, \varepsilon_{z x}$, and $\varepsilon_{z y}$ strains, wh ich are related to $\mathrm{Z}$-axis direction, as shown by Eqs. (8a) and (8b). These results show that the higher the centrifugal stress in the Z-axis direction, the more important the effect of stress-relaxation at the blade root.
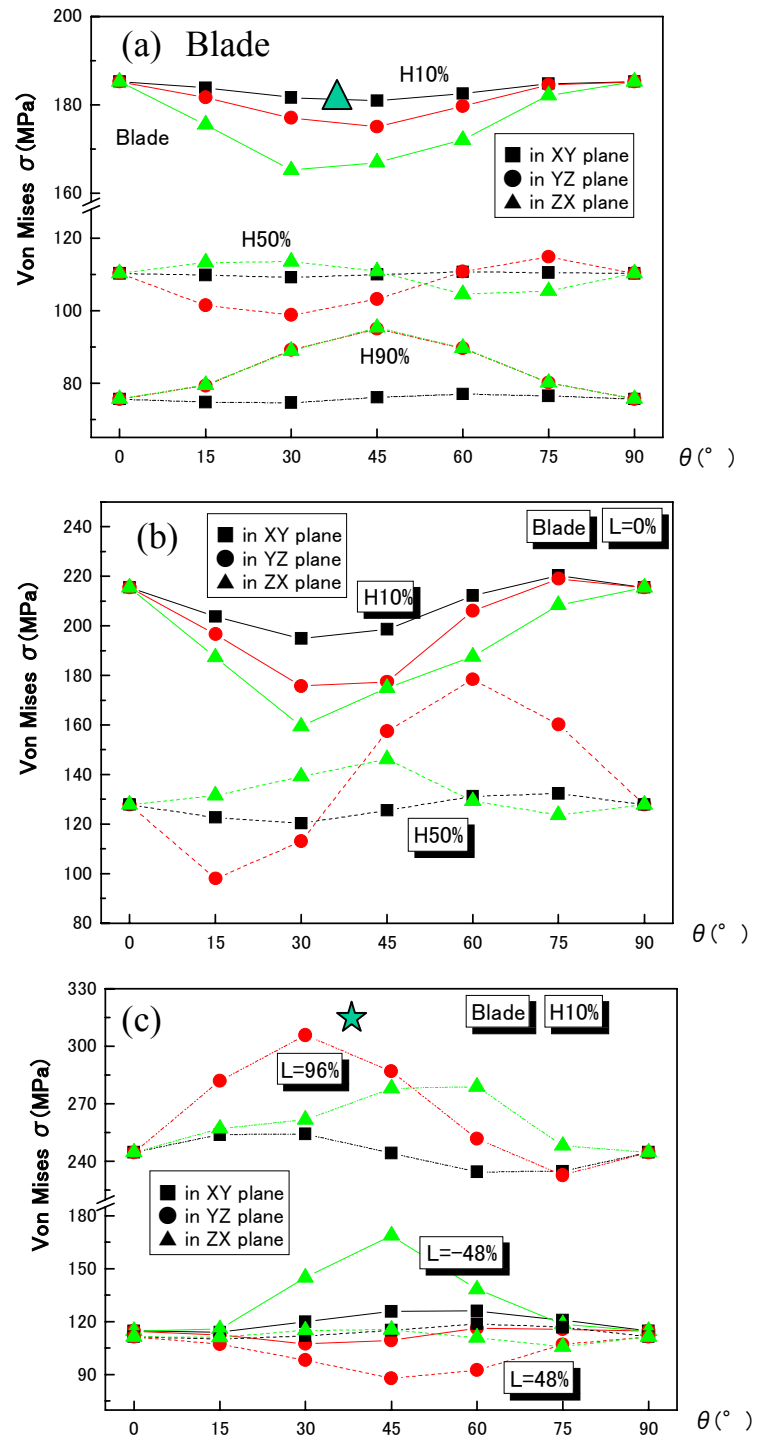

Fig. 4 Relationship between the Mises stress and $\theta$ for three axis of blade at the different spanwise cross section (a) and nodes (b), (c). 


\subsubsection{Creep rupture life}

A creep rupture life $t_{r}$ is estimated by Eq. (9) for each node. ${ }^{(13)}$.

$$
t_{r}=10^{\left(P_{f}-T C\right) / T}
$$

where, $T$ is the absolute temperature $(\mathrm{K}), C$ is a constant $(20)$, and $P_{\mathrm{f}}$ is the Larson Miller parameter. Strictly speaking, anisotropy of the Larson Miller curves of the SC superalloys should be considered, but in this analysis, the Larson Miller curve of the SC superalloys with [001] was used for all the direction as an approximation, as reported in introduction.

The relationship between the creep rupture life and $\theta$ for the turbine blade is shown in Figs. 5 (a)-(c). For the YZ in-plane, the creep rupture life was shorter for higher Mises stress and was strongly dependent on temperature. Therefore, although the stress at the blade trailing edge was maximum (Fig. 4 (c), L=96\%, 岤), the creep rupture life was shorter for the leading edge (Fig. 5 (b), $\mathbf{\Delta}$ ) than for the trailing edge (Fig. 5 (c), 行) because the temperature near the trailing edge was lower than near the leading edge (Fig. 2, two arrows and dashed line).

As shown in Fig. 5, the creep rupture life for the $\mathrm{H} 10 \%$ cross-section was the shortest of the three cross-sections. For the $\mathrm{H} 10 \%$ cross-section, it is clear that the creep rupture life for the XY in-plane was the shortest of the three coordinate planes (Fig. 5 (a), $\boldsymbol{\Delta})$. We reported that the leading edge, tip, and root of the turbine blade have a shorter creep rupture life under the same loads and boundary conditions as used in this analysis ${ }^{(3)}$. This means that, the creep rupture life of the root of a blade made of SC superalloy is the shortest, when the blades rotate with higher rotation speed.

Usually the design specifications of the most dangerous part of an object are taken as the design limitation for the entire object. This means that, if the creep rupture life of the blade root can be extended by making the $<100>$ crystallographic axis the $\mathrm{Y}$ or $\mathrm{X}$ axis (the $\mathrm{ZX}$ or YZ in-planes) of the blade, the creep rupture life of the
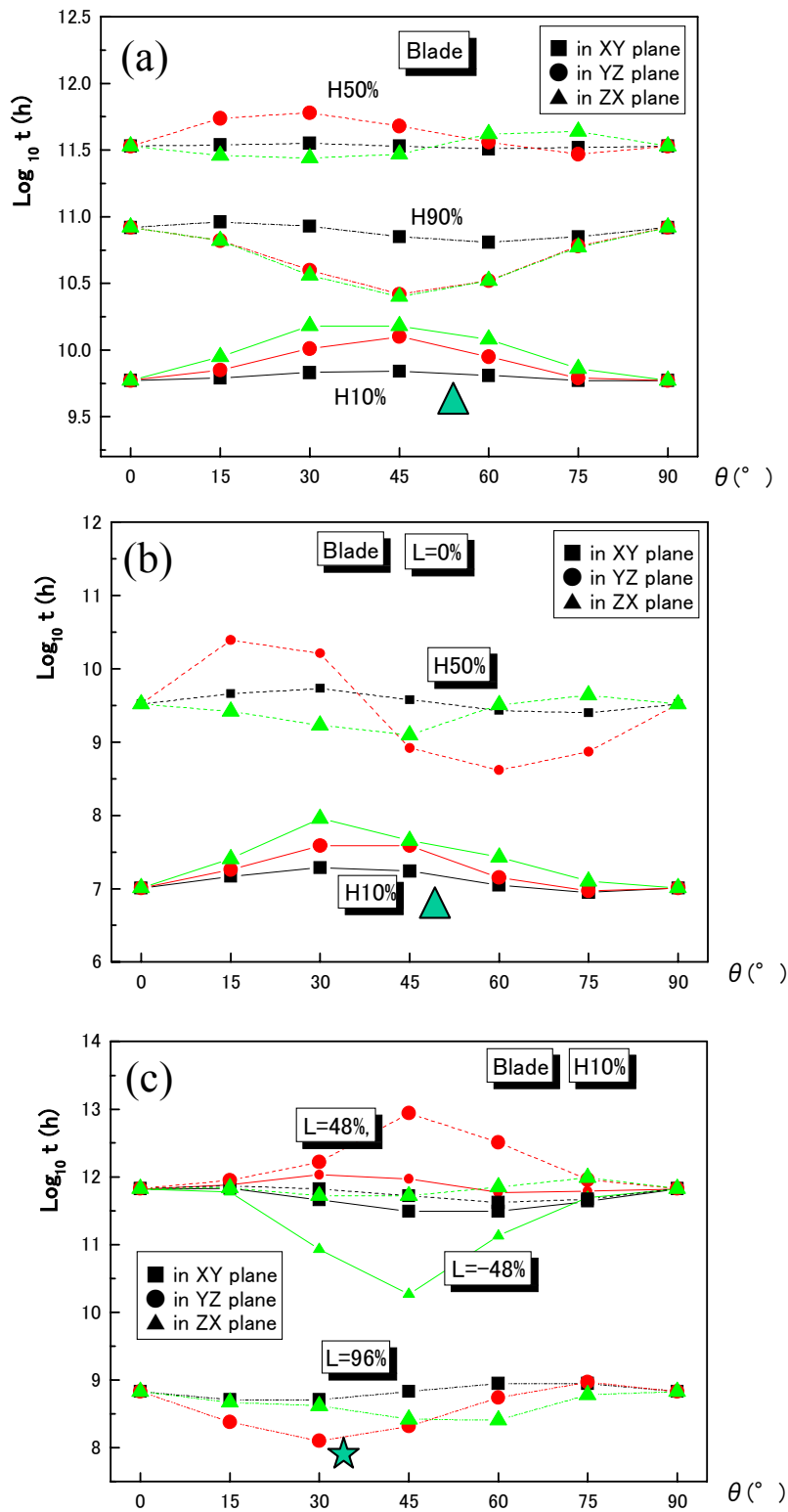

Fig. 5 Relationship between the creep rupture time and $\theta$ for three axis of blade at the different span-wise cross section (a) and nodes (b), (c). 
blade can be extended.

While making blades in which the $<100>$ crystallographic axis is the $\mathrm{Y}$ or $\mathrm{X}$ axis is a technological issue, the results of this analysis indicate that the best way to use the anisotropy is to control the growth axis of a SC to reconciling their property with the operating environment.

\section{Conclusions}

We have investigated the effects of the crystal orientations $(\theta)$ of the coordinate planes on the structural strength of SC turbine vanes and blades $\left(1400^{\circ} \mathrm{C}\right.$ class) made of TMS-75 SC superalloy. The results obtained in present work are:

(1) The elastic constant matrix changes were equivalence for each of three coordinate due to the orientation variation $\left(0^{\circ}<\theta<90^{\circ}\right)$, and the strength of the turbine vane and blade were strongly related to $\theta$, and also depended on the load and model shape.

(2) The dependence of the vane strength on the crystal orientation depended on the coordinate plane: the Mises stress was lower for the XY in-plane and maximum near $\theta=$ $45^{\circ}$ for both the $\mathrm{YZ}$ and $\mathrm{ZX}$ in-planes. It clear that the present control method of orientation on the actual vanes made of TMS-75 SC superalloy was appropriate.

(3) For a blade, the effects of the crystal orientations on blade tip were similar to the vane, but it was dissimilar for the blade root: the Mises stress was larger for the XY in-plane and minimum near $\theta=45$ and $30^{\circ}$ for the $\mathrm{YZ}$ and ZX in-planes, respectively. It is clear that the creep rupture time can be extended, when the $<100>$ crystallographic axes is the $\mathrm{Y}$ or $\mathrm{X}$ axis of the blade under higher rotation speed.

The anisotropies of creep deformation and plastic deformation should also be considered in the measurement of other material properties. The general effects of the crystal orientation, model shape, and load on the structural strength will be investigated in the next step of this research.

\section{Acknowledgments}

This research is a part of High Temperature Materials 21 Project of the National Institute for Materials Science, Japan. We would like to express our gratitude to Toshio Nishizawa of Japan Aerospace Exploration Agency for cooperation.

\section{References}

(1) Harada, H., Okaxaki, M., High temperature strength of NI-based superalloys and coatings for advanced gas turbines, Journal of the Society of Materials Science, Japan, Vol.51, No.7 (2002), pp.836-842 (in Japanese).

(2) Lambropoulos, J. C., The Isotropic assumption during the Czochralski Growth of Single Semiconductors Crystals, Journal of Crystal Growth, Vol.84, No.3 (1987), pp.349-358.

(3) Chen, J. et al., Structural Strength Change by Single Crystallization of Turbine Blades and Vanes, Journal of the Society of Materials Science, Japan, Vol.54, No.3 (2005), pp.251-256 (in Japanese).

(4) Miyazaki, N. et al., Elastic constant matrix required for thermal stress analysis of bulk single crystals during czochralski growth, Journal of Crystal Growth, Vol.106 (1990), pp.149-156.

(5) Miyazaki, N. et al., Thermal Stress Analysis of Bulk Single Crystals during CZ Growth: Anisotropic Effects in Various Single Crystals, Transactions of the Japan Society of Mechanical Engineers, Series A, Vol.58, No.554 (1992), pp.1942-1946 (in Japanese).

(6) Jordan, A. S., A Thermoelastic Analysis of Dislocation Generation in Pulled GaAs Crystals, The Bell System Technical Journal., Vol.59 (1980), pp.593-636.

(7) Miyazaki, N. et al., Thermal Stress Analysis of Bulk Single Crystal during Czochralski Growth: Comparison between Anisotropic Analysis and Isotropic Analysis, Transactions of the Japan 
Society of Mechanical Engineers, Series A, Vol.57, No.536 (1991), pp.858-863 (in Japanese).

(8) Ohno, N. et al., Multiaxial creep of a nickel-base directionally solidified alloy: anisotropy and simulation, Acta Materialia, Vol.40, No.3 (1992), pp. 559-567.

(9) Mizuno, T., Ohno, N., Anisotropy in multiaxial creep of a nickel-base single-crystal superalloy CMSX-2 ( $2^{\text {nd }}$ report, discussion based on 3D finite-element analysis), Transactions of the Japan Society of Mechanical Engineers, Series A, Vol.58, No.556 (1992), pp. 2446-2452 (in Japanese).

(10) Okazaki, M. et al., Nucleation of Cellular Colonies in Single Crystal Ni-Based Superalloy Subjected to Previous Damage and Influence on High Temperature Fatigue Strength, Journal of the Society of Materials Science, Japan, Vol.50, No.2 (2001), pp.94-100 (in Japanese).

(11) Hiyoshi, N., Sakane, M., Tension-Torsion Multiaxial Creep-Fatigue for CMSX-2 Nickel Base Single Crystal Superalloy, Journal of the Society of Materials Science, Japan Vol. 50, No.2 (2001), pp.137-143 (in Japanese).

(12) Yamamoto, M. et al., Evaluation of Crack Initiation and Propagation Lives from Internal Defects and Lower Limit of Failure Life in High Temperature Fatigue on Ni-Based Single Crystal Superalloy, Journal of the Society of Materials Science, Japan, Vol.50, No. 5 (2001), pp.510-515 (in Japanese).

(13) Chen, J. et al., Effects of the Cross-Sectional In-Plane Crystal Orientation on the Structural Strength of Single-Crystal Turbine Vanes, Journal of the Society of Materials Science, Japan, (In press).

(14) Chen, J. et al., Interface Construction for Thermal Stress Analysis in Virtual Turbine and Stress Evaluation, journal of the Gas Turbine Society of Japan, Vol.32, No.1 (2004), pp.34-39 (in Japanese).

(15) Zener. C. M., Elasticity and anelasticity of metals, The University of Chicago Press, (1948), p.7-22.

(16) Bunge H.J., The relation between preferred orientation and the Lankford parameter of plastic anisotropy, Arch. Eisenhuttenwes, Vol.52, No.10, (1981), pp.407-411.

(17) Washizu, K. et al., Finite Element Method Handbook, II applied compilation, the Baifukan Press (1983), pp.368-375 (in Japanese).

(18) Noda, N. et al., Fundamental Elastic Mechanics, the Nisshin Publication Press, (1988), pp.6-25 (in Japanese).

(19) Zako, M., Matsumoto, K., Behavior Analysis of Composite Material, Tokyo, Asakura book store, (1998), pp.14-21 (in Japanese). 